\title{
Illicit Drugs Breaking One Heart at a Time
}

\author{
Ayman Battisha ${ }^{1}$ \\ ${ }^{1}$ Department of Internal Medicine, University of Massachusetts- \\ Baystate, Springfield, Massachusetts, United States
}

\begin{abstract}
Address for correspondence Ayman Battisha, MD, Department of Internal Medicine, University of Massachusetts-Baystate, 700 Chestnut Street, Springfield, MA 01107, United States (e-mail: Ayman.BattishaMD@baystatehealth.org).
\end{abstract}

\begin{abstract}
Keywords

- angina

- cardiac output

- heart failure

- systolic dysfunction

Takotsubo cardiomyopathy (TCM) is triggered by multiple physical and psychological stressors and frequently mimics acute coronary syndrome. Acute alcohol intoxication as a trigger for TCM has been rarely reported as TCM is usually associated with alcohol withdrawal, not intoxication. The authors report a 41-year-old woman with polysubstance and alcohol abuse history, who presented with acute alcohol intoxication and electrocardiogram demonstrating ventricular tachycardia with syncope. Laboratory parameters revealed elevated Troponin-I and metabolic acidosis. Coronary angiogram was unrevealing for coronary atherosclerosis and she was managed conservatively for acute heart failure from TCM.
\end{abstract}

\section{Introduction}

Stress cardiomyopathy is a syndrome characterized by transient regional systolic dysfunction of the left ventricle (LV) mimicking myocardial infarction (MI), in the absence of obstructive coronary artery disease or plaque rupture. The incidence of Takotsubo's cardiomyopathy among individuals exposed to physical oremotional stress is unknown. It is unclear why postmenopausal women are affected disproportionately. We present a unique case of stress-induced cardiomyopathy following onset of polymorphic ventricular tachycardia.

\section{Case}

A 41-year-old woman with history of hypertension, third-degree heart block ( $\mathrm{s} / \mathrm{p}$ dual-chamber pacemaker [PPM]), and polysubstance disorder and/or alcohol use disorder was admitted due to acute alcohol intoxication. She was found to have a 9-second run of polymorphic ventricular tachycardia with syncope in the context of prolonged QT, with hypokalemia and hypomagnesemia. The next day, she complained of "fluttering" in her throat. Electrocardiogram (ECG) showed diffuse T-wave inversions, troponin spike of 1.9 , with admission troponins being negative. Echocardiogram showed akinesis of apical segments consistent with left anterior descending coronary artery (LAD) infarct or Takotsubo's cardiomyopathy with depressed LV function and ejection fraction (EF) 35 to 40\%, with prior left anterior ejection fraction (LVEF) being normal. Left heart catheterization showed no evidence of luminal narrowing consistent with a diagnosis of Takotsubo's cardiomyopathy. Repeat echocardiogram 3 weeks after discharge showed an LVEF of $62 \%$, exemplifying the reversible nature of Takotsubo's cardiomyopathy ( - Fig. 1).

\section{Discussion}

The incidence of Takotsubo's cardiomyopathy related to ventricular tachycardia and alcohol use is unknown. Most cases are related to withdrawal rather than acute alcohol intoxication, as in our case. However, prolonged QT is well reported in Takotsubo's cardiomyopathy, but actual torsades de pointes is rare. The exact incidence of Takotsubo's cardiomyopathy in premenopausal women is unknown; however, a meta-analysis on 6,178 cases showed women aged $>55$ years had 4.8 times higher odds for developing Takotsubo's cardiomyopathy when compared with women aged $<55$ years. Multiple reports suggest estrogen deficiency as a pathologic link, as estrogen exerts cardioprotective effects (inhibition of excessive sympathetic tone and RAAS [renin-angiotensinaldosterone system] activation). Ueyama et $\mathrm{al}^{1}$ reported that increased serum estradiol can diminish pathologic changes in the heart induced by stress. This case highlights that there is a large area for improvement in our understanding of this multicomplex disease.
License terms

(ㅇ) (1) $\ominus \circledast$ 


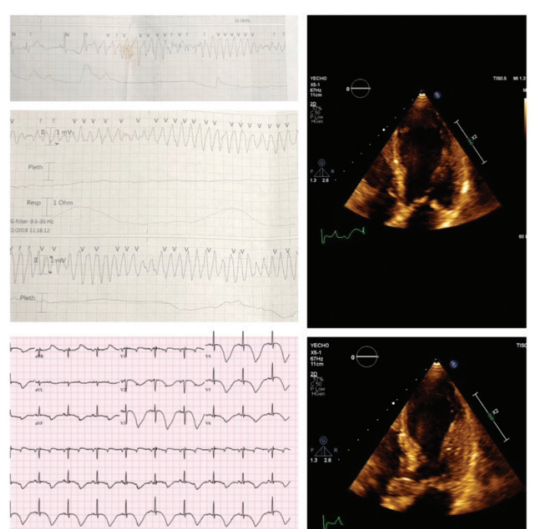

Fig. 1 Electrocardiogram showing run of polymorphic ventricular tachycardia and diffuse T-wave inversions, echocardiogram showing akinesia of apical segments.

\section{Reference}

1 Ueyama T, Hano T, Kasamatsu K, Yamamoto K, Tsuruo Y, Nishio I. Estrogen attenuates the emotional stress-induced cardiac responses in the animal model of Tako-tsubo (Ampulla) cardiomyopathy. J Cardiovasc Pharmacol 2003;42 (Suppl 1):S117-S119 